\title{
The effect of observing different information on learning the basketball jump shot
}

\author{
Saleh Rafiee ${ }^{1, *}$ and Amir Dana ${ }^{2}$ \\ ${ }^{I}$ Department of Motor Behavior, Sport Science Research Institute of Iran, Tehran, Iran; and ${ }^{2}$ Department of Physical Educa- \\ tion, Tabriz Branch, Islamic Azad University, Tabriz, Iran
}

Copyright: (C) 2019 S. Rafiee and A. Dana. This is an open access article licensed under the Creative Commons Attribution License (https://creativecommons.org/licenses/by/4.0/).

\begin{abstract}
Background: Absolute and relative information may have different roles during motor learning through observation. Objective: This study aimed to address the effect of observing different information on the learning of the basketball jump shot. Methods: For this purpose, 30 participants were included in the study and divided into three groups (Full Body, Arm, and Wrist) according to the information they observed. Full Body group observed information relating to all body parts, Arm group observed information relating to the throwing arm, and the Wrist group observed the information relating to the throwing wrist. All information was presented in the point light format. During data acquisition, participants performed 30 trials which were observed in the model 5 times before the first trial and once before the remaining trials. Twenty-four hours later, 10 trials were performed as a retention test. After retention, there was a re-acquisition period in which all groups observed full-body information in an order similar to the acquisition period and then performed 10 trials. Accuracy, visual search (eye fixations), and kinematics data (intra-limb coordination variability) were acquired. Results: No between-group difference existed in the accuracy measurement. Results of the kinematics data (intra-limb coordination) showed that Full Body and Arm groups performed more like the model than Wrist group. Visual search data showed that all groups were fixed more on the throwing arm than other parts of the body. Conclusions: These results showed that restricted relative information can be more useful for learning a throwing action.
\end{abstract}

Keywords: observational learning, point light display, perceptual degrees of freedom, visual perception perspective, intra-limb coordination

\section{Introduction}

Observational learning is a process by which observers watch the behavior of a model and adapt their movement as a result (Williams, Davids, \& Williams, 1999).

Researchers believe that observation creates a mental representation in the person's memory, which can later be retrieved and used to guide their behavior (Bandura, 1977). Although Bandura did not explain what type of information is used to construct such representations, other studies have suggested that the observer mainly uses relative motion information to learn a demonstrated skill (Scully \& Newell, 1985). It is believed that movement is identified by the observer through the motions of individual elements relative to each other. This was examined by comparing the video recording of a task with its point-light displays (PLD). PLD is a video

\footnotetext{
* Address for correspondence: Saleh Rafiee, Department of Motor Behavior, Sport Science Research Institute of Iran, Tehran, Miremad Street, 21452, Iran. E-mail: saleh_rafiee@yahoo.com
}

in which the structural information (texture) is removed and the information about reflexive markers attached to the body is displayed. It is believed that in PLD structural information is omitted and relative motion information is highlighted, which makes the detection of movement easier (Johansson, 1973). Therefore, it can be concluded that relative motion information plays an important role in observational learning. Using this approach, Romack (1995) showed that demonstrating basketball dribble in point-light format leads to better learning compared to video displays. Also, Scully and Carnegie (1998) found that there was an improvement in learning ballet movements with the point-light model compared to a video-recorded demonstration.

Despite these findings, the results of later studies have been inconsistent with these findings. For example, it has been shown that point-light demonstrations do not lead to better learning in a dart aiming task compared to normal video demonstrations (Al-Abood, Davids, Bennett, Ashford, \& Marin, 2001). Also, there 
were not observed significant differences in learning a soccer kick between point-light display and video groups (Horn, Williams, \& Scott, 2002; Horn, Williams, Scott, \& Hodges, 2005). In addition, Ghorbani and Bund (2016) found no difference between the effect of pointlight and video displays on leaning a baseball throw.

One of the problems with previous studies in this area is that the relative motion information has not been directly manipulated. The relative information considered as point light displays, however, the point light displayed can be manipulated and remove some of the body parts information. Hodges, Hayes, Breslin, and Williams (2005) attempted to examine the effect of manipulating relative motion in a soccer chipping task. The participants received three types of motion information: the lower leg (i.e., intra-limb relative motion), the foot (ankle and toe), or the toe (i.e., no relative motion). The results showed that the group receiving absolute motion information outperformed the other groups. These were the first findings to contradict the visual perception perspective (Scully \& Newell, 1985). In contrast, Hayes, Hodges, Huys, and Williams (2007) studied observational learning with a bowling task and found that the group observing end-points information outperformed the group observing the whole-body action. It was concluded that observing end point demonstrations is sufficient for learning a task and that observing every relative motion information may undermine learning due to higher information load.

Some studies have shown that, instead of relative and absolute motion information, limited relative motion information plays a major role in observational learning. Breslin, Hodges, Williams, Curran, and Kremer (2005) examined the importance of relative motion information in a cricket bowling task. They found that observing a point-light demonstration does not necessarily result in superior motor performance and learning over classic video demonstration. In Breslin, Hodges, and Williams (2009), another group was added that observed the relative motion information from the model's bowling arm. The results showed better coordination learning in this group, and visual search data indicated that observers mainly focused their gaze on the model's bowling arm. Thus, it was concluded that information from the bowling arm is the most important source of information for learning a cricket bowling task.

According to the visual perception perspective (Scully \& Newell, 1985), relative motion information is important only at the early stages of the learning process. There are not many studies on this prediction of visual perception perspective. Hayes et al. (2007) assigned participants to a relative information group that observed the whole-body action and a limited information group that observed the endpoints of a bowling task. They believed that if relative motion information is useful at the early stages of learning, providing relative motion information at the end of a training period with absolute motion information should not have a significant effect on learning. They showed that relative information had no effect on coordination, but improved accuracy in the end-point group. In another study that was aimed at examining the visual perception perspective, Breslin et al. (2009) found that full-body information did not affect coordination, but improved visual search.

The literature review reveals the inconsistencies between different studies. A possible reason is the use of simple tasks that already exist in the individual's motor repertoire (Al-Abood et al., 2001; Breslin et al., 2009; Hodges et al., 2005) or tasks that do not require full-body motion (Hodges et al., 2005). In addition, the majority of the aforementioned studies, except Breslin et al. (2009) did not use a visual search system and did not directly examine the type of information used during observing the model. Therefore, the present research tries to contribute to the literature by using a task that requires full-body coordination and by examining eye movements during observation. According to the visual perception perspective (Scully \& Newell, 1985), it was hypothesized observing relative information would results in outperformance in retention tests than observing absolute information. Also, it was hypothesized that the visual search of the participant would be focused on relative information instead of absolute information.

\section{Methods}

\section{Participants}

Thirty males (age $27.4 \pm 2.1$ years, height $178 \pm 12$ $\mathrm{cm}$, weight $75 \pm 8 \mathrm{~kg}$ ) participated in this study. Participants reported no prior experience in playing the basketball game. They were all right-handed and had normal or corrected to normal vision. Based on selfreports, the participants had no history of neural disorder that would affect coordination or motor control. Also, an informed consent form was filled by participants before taking part in this research. This study was approved by the research ethics committee of the Sport Science Research Institute of Iran. All of the stages (acquisition, retention, and re-acquisition) were performed in the laboratory.

\section{Task}

The task was the basketball jump shot, performed when standing behind the free-throw line. 


\section{Data collection}

An expert with 10 years of experience in professional basketball (center player), currently playing in the Iranian Basketball Super League, was asked to help create the model in laboratory settings. To create the pointlight pattern and record the movement's kinematic information, 17 reflective markers were placed on the distal head of the 5th metatarsal (toe), the lateral malleolus (ankle), the lateral condyle of the femur (knee), the greater trochanter (hip), the acromion process (shoulder), the lateral epicondyle (elbow), the styloid process of radius (wrist), the metacarpal head (index finger), and the center of the forehead; markers were placed on both sides of the body (Breslin et al., 2005; Hayes et al., 2007). After a warm-up (selected according to the model's preferred routine), the model was asked to stand at the throwing line and perform the jump shot (no instructions provided for the expert to perform this task). One of the attempts in which the ball passed through the basket without touching the backboard and rim was recorded and used as the model film. This film was edited into three videos with equal duration using a Simi Motion software (Version 7.5.305; SIMI Reality Motion Systems, Unterschleißheim, Germany): a video of the whole point lights placed on the body (relative information - Full Body group), a video of the four markers placed on the throwing arm (limited relative information - Arm group), and a video of the marker placed on the wrist of the throwing arm (absolute information - Wrist group).

In the acquisition period, the participants were individually called to the laboratory and asked to complete a consent form while getting acclimatized with the environment. Then, the reflective markers were placed on their bodies similar to the model and the participants were asked to wear an eye-tracking device (SensoMotoric Instruments, Teltow, Germany). Before displaying the model video, the participants were informed of the specifics of the task and that it had been performed by an expert. They were also asked to equally focus on getting the ball through the basket and imitating the movement pattern. The model film was demonstrated to the participants five times before they performed their first jump shoot and once before each subsequent attempt. Each individual performed 30 attempts (three blocks of 10 attempts with one-minute rest between blocks) in the acquisition stage. During observation, eye movement patterns were recorded. Also, a SIMI motion analysis system (six cameras with $200 \mathrm{~Hz}$ sample rate; Version A406k; Basler, Ahrensburg, Germany) was used to record movement kinematics. It is important to note that the participants were randomly assigned to three groups based on the information they observed: Full Body, Arm, and Wrist.
Twenty-four hours later, the participants were again called to the laboratory, reflective markers were placed on their body, and each participant wore eye-tracking device to match the conditions of retention stage with those of the acquisition stage. For retention, each participant completed 10 attempts, but no film was demonstrated to the participants.

There was a re-acquisition period after the retention stage in which full-body information was presented to the participants in order to examine whether observing relative information after a training period affects motor learning. If relative motion information had any role in this stage of learning, it would improve coordination in the group that has not observed this information (the group observing the wrist movement). Similar to the acquisition, the participants performed 10 attempts, observed the film 5 times before the first attempt and once before each subsequent trial.

\section{Variables}

A scoring system $0-5$ was used to measure performance (accuracy) during acquisition and retention as follows (Aiken, Fairbrother, \& Post, 2012): a score of 5 if the ball passed through the basket without touching the rim, 4 if the ball went through the basket after touching the rim or the backboard, 3 if the ball touched the rim only, 2 if the ball touched the rim and backboard and does not pass the basket, 1 if the ball touched the basket only, and 0 if the ball touched neither.

A comparison of the intra-limb coordination (shoulder-elbow, and wrist-elbow) of the participants with that of the model was made using Normalized root-meansquare error (NoRMS). This measure provides the similarity of two joints of the participant (for example shoulder and elbow) in relation to the two joints of the model (Hodges et al., 2005). This measure is a method to quantify the angle to angle plots. NoRMS is a modified version of the procedure proposed by Sidaway, Heise, and Schoenfelder-Zohdi (1995). It provides a measure of similarity to the model's performance, with lower values indicating greater similarity (Hayes et al., 2007; Hodges et al., 2005). Since all the participants were right-handed and the task was performed with the right hand, the kinematics of the right side of the body was compared to those of the model. These included elbow-wrist coordination. The first flexion in the elbow was considered as the starting point and maximum extension after the jump shot was considered as the ending point of the movement. The data were smoothened with a recursive 4 th order Butterworth filter, with a cutoff frequency of $7 \mathrm{~Hz}$, and a linear interpolation was performed to normalize the data to 100 points (Winter, 1990). During acquisition, kinematic data from the first three trials of the first block (attempts 1-3), the last 
three trials of the first block (attempts 8-10), the last three trials of the second block (attempts 18-20), and the last three trials of the third block (attempts 28-30) were used to calculated NoRMS (Breslin, Hodges, Williams, Kremer, \& Curran, 2006; Breslin et al., 2009). For the retention and re-acquisition stages, the first three trials (attempts 1-3) and the last three trials (attempts 8-10) were analyzed.

As for visual search, data from the first five observations in the acquisition and re-acquisition blocks were used for analysis. Gaze data was analyzed using BeGaze Software (SensoMotoric Instruments, Teltow, Germany). The fixation number, percentage of fixation time, and the mean of the fixation time were calculated. An area of interest was assigned to each body part (throwing arm, feet, and wrist) to determine the fixation number and fixation duration for each area. To examine whether the demonstration of limited or complete information releases the perceptual degrees of freedom (Breslin et al., 2009), the number of fixations on limited areas (in groups with limited information and full-body information) was calculated, so that later the frequency and duration of fixations in the acquisition stage and the re-acquisition stage could be compared.

\section{Statistical analysis}

Shapiro-Wilk was used to test the normality of data. A 3 (model type: Full Body, Arm, and Wrist) $\times 4$ (acquisition blocks) mixed-design analysis of variance (ANOVA) with repeated measures on the last factor was used to analyze the accuracy data during acquisition. A 3 (model type: Full Body, Arm, and Wrist) $\times 2$ (retention blocks) mixeddesign analysis of variance with repeated measures on the last factor was used to compare groups in the retention stage. Also, a 3 (model type: Full Body, Arm, and Wrist) $\times 3$ (re-acquisition blocks and final acquisition block) mixed-design analysis of variance with repeated measures on the last factor was used to compare groups in the re-acquisition stage and to determine whether demonstrating the model in the re-acquisition stage had any effect on the accuracy of the participants. There was similarity between the statistical analysis for kinematics data and the accuracy data in all periods. All statistical analyses were performed by SPSS software (Version 16; SPSS, Chicago, IL, USA).

Data related to the percentage of fixating time on the area of interest was averaged for each individual in each block, the results of which were analyzed using a 3 (model type, Full Body, Arm, and Wrist) $\times 3$ (fixation points: throwing arm, feet, and wrist) $\times 2$ (tests) mixeddesign analysis of variance with repeated measures on the last factor. Data related to the number of fixations was averaged for each individual in each block, the results of which were analyzed using a 3 (model type:
Full Body, Arm, and Wrist) $\times 2$ (tests) mixed-design analysis of variance with repeated measures on the last factor. Finally, the mean fixation duration was analyzed using a 3 (model type: Full Body, Arm, and Wrist) $\times 2$ (tests) mixed-design analysis of variance with repeated measures on the last factor. The partial eta squared $\left(\eta_{\mathrm{p}}^{2}\right)$ was calculated as effect size. The Bonferonni test was used as post-hoc test. All statistical analysis was performed at the .05 significance level.

\section{Results}

\section{Movement accuracy}

Figure 1 shows the accuracy data for each group in different stages. The results of mixed ANOVA for the acquisition stage showed that the main effect of group was not significant, but the main effect of the block was significant $\left(F(3,81)=6.13, p=.001, \eta_{\mathrm{p}}^{2}=.07\right)$. Moreover, the group-block interaction effect was not significant. The Bonferroni post-hoc test showed a significant difference between the first block and the other blocks $(p=.02)$, but no significant difference between the other blocks $(p=.21)$. Mean comparisons indicated that the participants had higher accuracy in second, third, and fourth blocks compared to the first block (means: 1 st block $=1.68,2$ nd block $=1.95,3$ rd block $=2.00,4$ th block $=1.99$ ).

In the retention stage, the results of ANOVA showed that the main effect of group was not significant. Also, the main effect of block and the group-block interaction effect were not significant.

In the re-acquisition, the results of mixed ANOVA showed that the main effect of group was not significant. However, the main effect of the block was significant $\left(F(2,54)=4.63, p=.01, \eta_{\mathrm{p}}^{2}=.14\right)$. In addition, the group-block interaction effect was not significant. The results of the Bonferroni post hoc test showed a significant difference between the last acquisition block and the first re-acquisition block $(p=.03)$. Also, a significant difference existed between the first and second re-acquisition blocks $(p=.02)$. However, the difference between the fourth acquisition block and the second re-acquisition block was not significant $(p=.62)$. Mean comparisons showed that the participants had higher accuracy in the last acquisition block and the second re-acquisition block compared to the first re-acquisition block (means: 4th acquisition block $=1.99,1$ st re-acquisition block $=1.79,2$ nd reacquisition block $=2.01$ ).

\section{Elbow-wrist coordination}

Figure 2 shows the elbow-wrist coordination data for each group in different stages. The results of mixed 


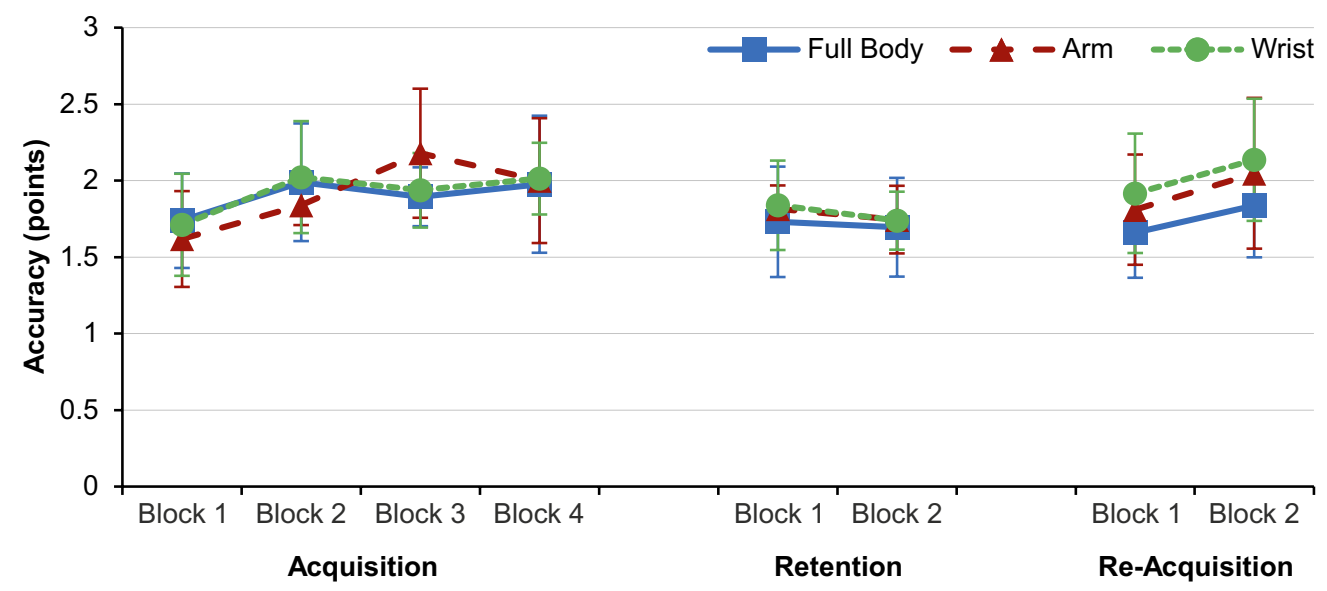

Figure 1. The accuracy in different stages. Error bars represent standard deviations.

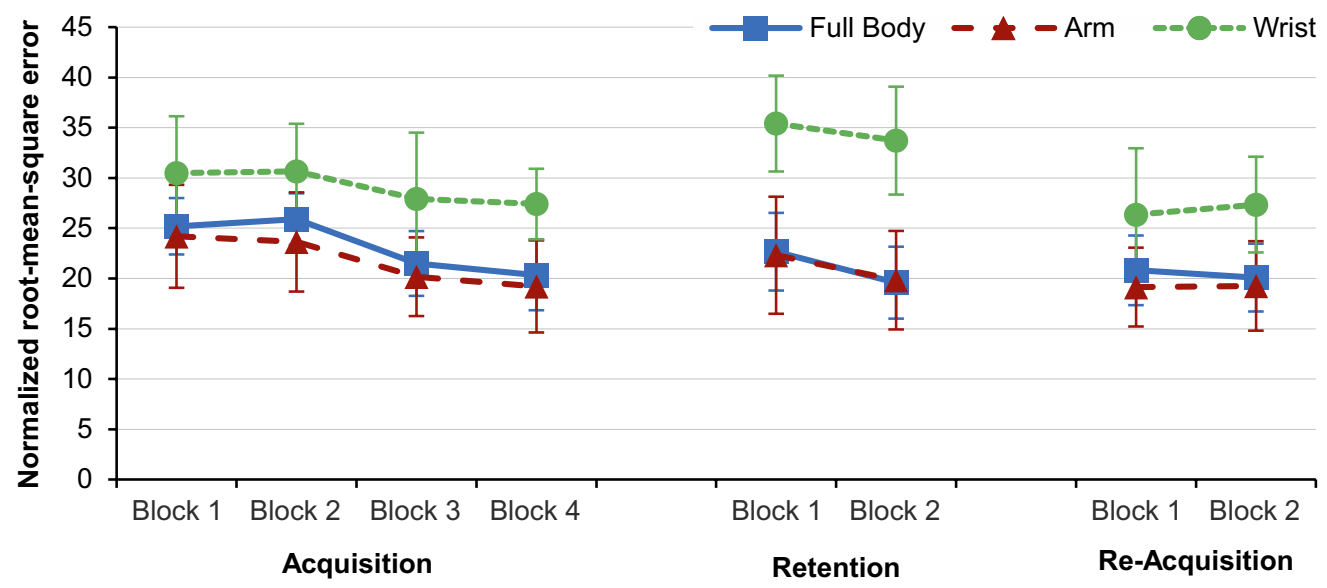

Figure 2. Elbow-wrist coordination in different stages. Error bars represent standard deviations.

ANOVA for elbow-wrist coordination in the acquisition showed that the main effect of group was significant $\left(F(2,27)=3.78, p=.03, \eta_{\mathrm{p}}^{2}=.21\right)$. The results also showed that the main effect of block was significant $\left(F(3,81)=11.05, p<.001, \eta_{p}^{2}=.29\right)$, but the groupblock interaction effect was not significant. The Bonferroni test was performed for the main effect of group and the results showed a significant difference between the Full Body and the Wrist groups $(p=.01)$. Also, a significant difference existed between the Arm and the Wrist groups ( $p=.02$ ), but the difference between the Full Body and the Arm group was not significant $(p=.37)$. Mean comparisons indicated that the Full Body and Arm groups had less variability in elbow-wrist coordination compared to the Wrist group (means: Full Body $=23.22$, Arm $=21.8$, Wrist $=29.12$ ) .

The results of the Bonferroni test for the main effect of block showed no significant difference between the first and second blocks $(p=.76)$. However, these two blocks were significantly different from the third and fourth blocks (all $p<.05$ ). Mean comparisons showed that the groups had less variability in their elbow-wrist coordination in the third and fourth blocks compared to the first and second blocks (means: 1st block $=26.62,2$ nd block $=26.73,3$ rd block $=23.19$, 4 th block $=22.31$ ).

In the retention, the results of mixed ANOVA showed that the main effect of the group $(F(2,27)=7.92$, $\left.p=.002 ; \eta_{\mathrm{p}}^{2}=.37\right)$ was significant. The main effect of block was also significant $(F(1,27)=24.70, p<.001$, $\left.\eta_{\mathrm{p}}^{2}=.56\right)$. However, the interaction effect of these two factors was not significant. The results of the Bonferroni post-hoc test for the main effect of the group showed that the Full Body and Arm groups were significantly different than Wrist group (all $p<.05$ ). However, the difference between Full Body and Arm groups was not significant $(p=.84)$. Mean comparisons showed that the Full Body and Arm groups had less variability in elbow-wrist coordination from the Wrist group (means: Full Body $=21.12$, Arm $=21.06$, Wrist $=34.56$ ). As for the main effect of block, mean comparisons showed that groups had less variability in their movement 
pattern in the second retention block compared to the first retention block (means: 1 st block $=26.79,2$ nd block $=24.37)$.

The results of mixed ANOVA for the re-acquisition showed that the main effect of group was significant $\left(F(2,27)=3.31, p=.05, \quad \eta_{\mathrm{p}}^{2}=.19\right)$. However, the main effect of the block and group-block interaction were not significant. The Bonferroni test for the main effect of group showed a significant difference in the Full Body and Arm groups than the Wrist group (all $p<.05)$. However, the difference between the Full Body and Arm groups was not significant $(p=.63)$. Mean comparisons showed lower variability in elbowwrist coordination in the Full Body and Arm groups than the Wrist group (means: Full Body $=20.40$, Arm $=19.20$, Wrist $=27.03)$.

\section{Percentage of fixating time on different locations}

Figure 3 shows the percentage of fixation time at different locations. The results of ANOVA showed that the main effect of group was significant $(F(2,27)=47.32$, $\left.p<.001, \eta_{\mathrm{p}}^{2}=.77\right)$. Also, from the results the main effect of fixation point $(F(2,27)=10.99$, $\left.p<.001, \eta_{\mathrm{p}}^{2}=.97\right)$ and the main effect of test time $\left(F(2,27)=22.23, p<.001, \eta_{\mathrm{p}}^{2}=.45\right)$ were significant. Moreover, the interactions between group and fixation point $\left(F(4,54)=433.75, p<.001, \eta_{\mathrm{p}}^{2}=.97\right)$, group and test time $\left(F(2,27)=70.37, p<.001, \eta_{\mathrm{p}}^{2}=.83\right)$, fixation point and test time $(F(2,54)=529.38$, $\left.p<.001, \eta_{\mathrm{p}}^{2}=.95\right)$, and group and fixation point and test time $\left(F(4,54)=10.90, p<.001, \eta_{\mathrm{p}}^{2}=.98\right)$ were significant. The Bonferroni test for the main effect of group showed a significant difference in the Full Body and Arm groups from the Wrist group (all $p<.05$ ). Mean comparisons indicated that the Full Body and Arm groups had higher percentage of fixaton time than the Wrist group (means: Full Body $=27.96$, Arm $=28.39$, Wrist $=23.59$ ). The Bonferroni test for the effect of fixation location showed that all the fixation locations were significantly different (all $p<.05$ ). Means comparisons indicated that groups fixated more on the throwing arm, respectively by the wrist and feet, respectively (means: throwing arm $=44.26$, wrist $=24.02$, feet $=11.66$ ). For the main effect of test time, mean comparisons showed that the percentage of fixation time was higher in the acquisition than the re-acquisition (means: acquisition $=27.52$, re-acquisition $=25.76$ ) .

The post-hoc test was performed for interaction effects. Since the three-way interaction of the factors were significant and performing the post-hoc test for this effect also involved other interactions, the results were not provided to prevent repetition. The post-hoc test for the interaction of the group, fixation location, and test time showed no significant difference between the pretest and post-test in the percentage of fixation time on different locations in the Full Body group (all $p<.05$ ). In this group, a significant difference was found between the percentage of fixation at different locations during acquisition and re-acquisition (all $p<.05$ ). Mean comparisons indicated that the participants fixated more on the throwing arm in both acquisition and re-acquisition stages, followed by the wrist and the feet, respectively (means in acquisition: $\operatorname{arm}=55.69$, feet $=12.72$, wrist $=15.47$; means in reacquisition: $\operatorname{arm}=55.27$, feet $=13.24$, wrist $=15.38$ ) . Moreover, the results of the post-hoc test showed that in the Arm group, a significant difference existed between the pretest and posttest in the percentage of fixation time of different locations (all $p<.05$ ). Mean comparisons showed that, in this group, the percentage of fixation time on the throwing arm was higher in the

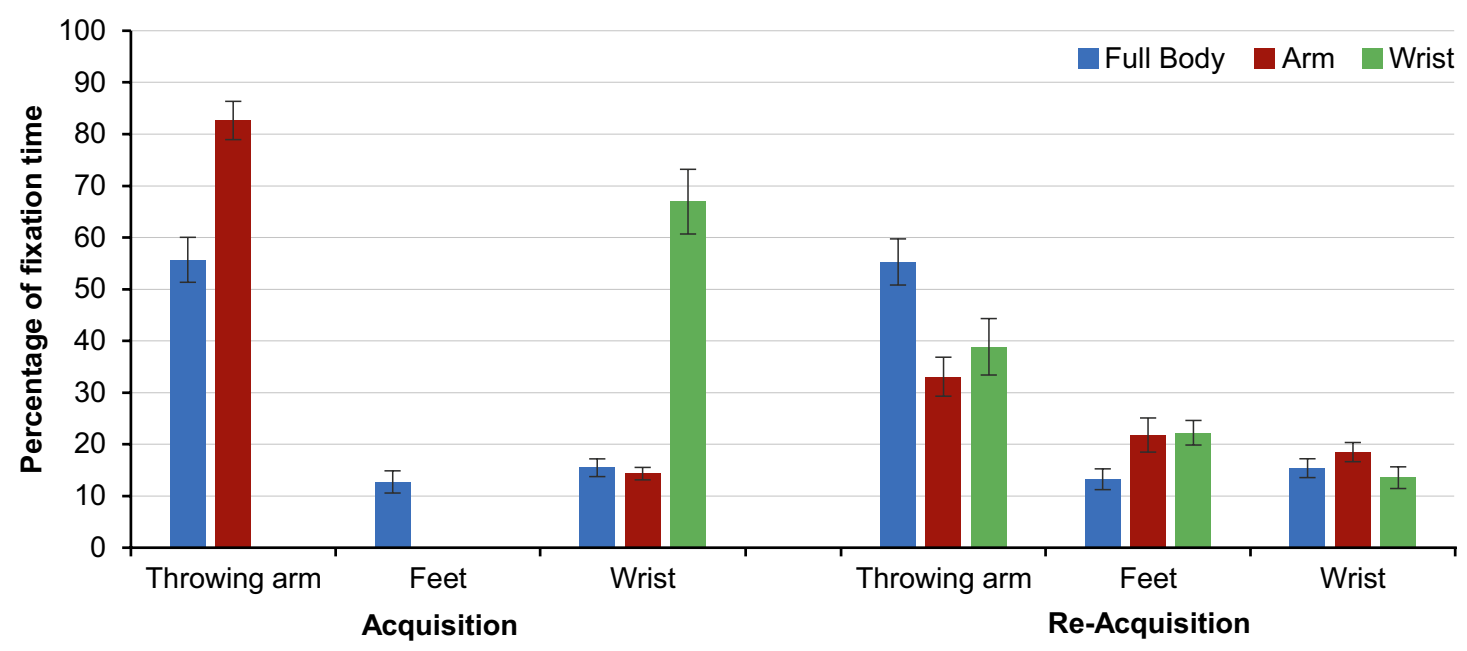

Figure 3. Percentage of fixation time on different locations. Error bars represent standard deviations. 
acquisition compared to the re-acquisition. Moreover, the results showed that in this group, the percentage of fixation time on the feet was higher in the re-acquisition stage than the acquisition. The same was true for the Wrist. In this group, the percentage of fixation time on the feet was higher than the wrist during reacquisition (means: arm in acquisition $=82.64$, arm in re-acquisition $=33.08$, feet in acquisition $=0$, feet in re-acquisition $=21.79$, wrist in acquisition $=14.32$, wrist in re-acquisition $=18.49$ ). For the Wrist group, the results showed a significant difference between the acquisition and re-acquisition stages in the percentage of fixation time on different locations (all $p<0.05$ ). Mean comparisons showed that this group fixated more on the wrist in the acquisition stage, but during retention the percentage of fixation time on the throwing arm was higher, followed by the feet and the wrist, respectively (means: arm in acquisition $=0$, arm in re-acquisition $=38.86$, feet in acquisition $=0$, feet in re-acquisition $=22.23$, wrist in acquisition $=66.94$, wrist in re-acquisition $=13.54$ ). No other interaction effect was observed.

\section{Fixation number}

Figure 4 shows the number of fixations for each group in different test times. The results of mixed ANOVA showed that the main effect of the group was significant $\left(F(2,27)=86.16, p<.001, \eta_{\mathrm{p}}^{2}=.86\right)$. Also, from the results, the main effect of test time was significant $\left(F(1,27)=70.07, p<.001, \eta_{\mathrm{p}}^{2}=.72\right)$. However, the interaction of group and test stage was not significant $\left(F(2,27)=2.23, p=.12, \eta_{\mathrm{p}}^{2}=.14\right)$. The results of the post-hoc Bonferroni test for the main effect of the group showed that between-group differences were all

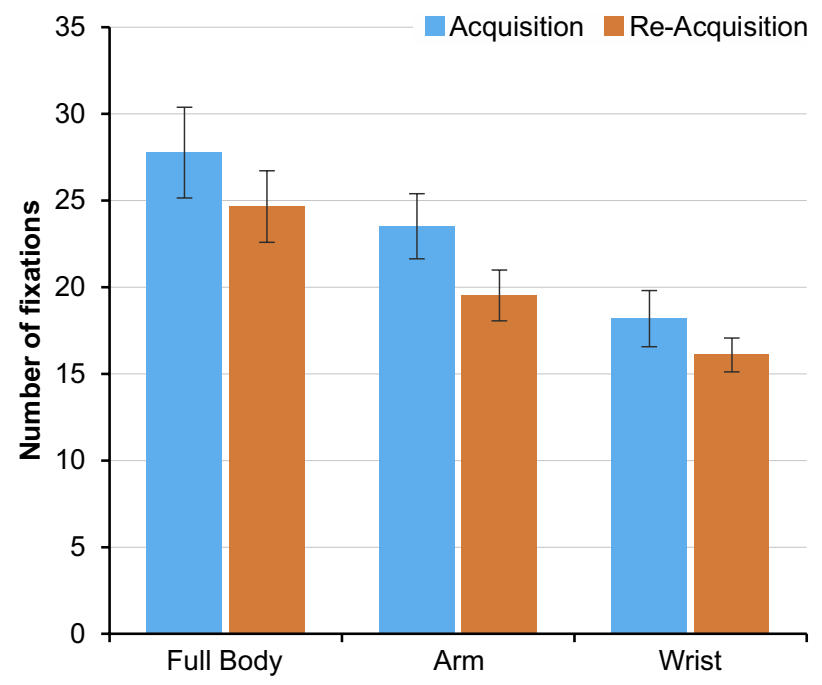

Figure 4. Number of fixations in different test times. Error bars represent standard deviations. significant (all $p=0.05$ ). Mean comparisons showed that the number of fixations was higher in the Full Body group than the other two groups and higher in the Arm group compared to the Wrist group (means: Full $\quad$ Body $=26.20, \quad$ Arm $=21.51, \quad$ Wrist $=17.13)$. Moreover, mean comparisons for the main effect of test time showed that groups had fewer fixations in re-acquisition than in the acquisition (means: acquisition $=23.15$, re-acquisition $=20.08$ ) .

\section{Mean of fixation duration}

Figure 5 shows the mean of fixation duration. The results of mixed ANOVA showed that the main effect of group was significant $(F(2,27)=3.97, p=.03$, $\left.\eta_{\mathrm{p}}^{2}=.71\right)$. Moreover, the results showed that the main effect of test time was significant $(F(1,27)=69.07$, $\left.p<.001, \eta_{\mathrm{p}}^{2}=.71\right)$. The interaction of group and test time was also significant $(F(2,27)=3.41, p=.04$, $\left.\eta_{\mathrm{p}}^{2}=.20\right)$. For the main effect of the group, the results of the Bonferroni post-hoc test showed that the Full Body group was significantly different from the other two groups (all $p<.05$ ). However, there was no significant difference between the Arm and the Wrist groups $(p=.67)$. Mean comparisons indicated that fixation duration was less in the Full Body group than the other two groups (means: Full Body $=2.42, \operatorname{Arm}=3.23$, Wrist $=2.85)$. For the interaction of group and test time, the results of post-hoc test showed that the Full Body group was significantly different from the other groups only in the acquisition (all $p<.05$ ). Mean comparisons indicated that the Full Body group had less fixation duration in the acquisition compared to the other two groups (means: Full Body $=2.89$, Arm $=4.33$, Wrist $=4.22$ ).

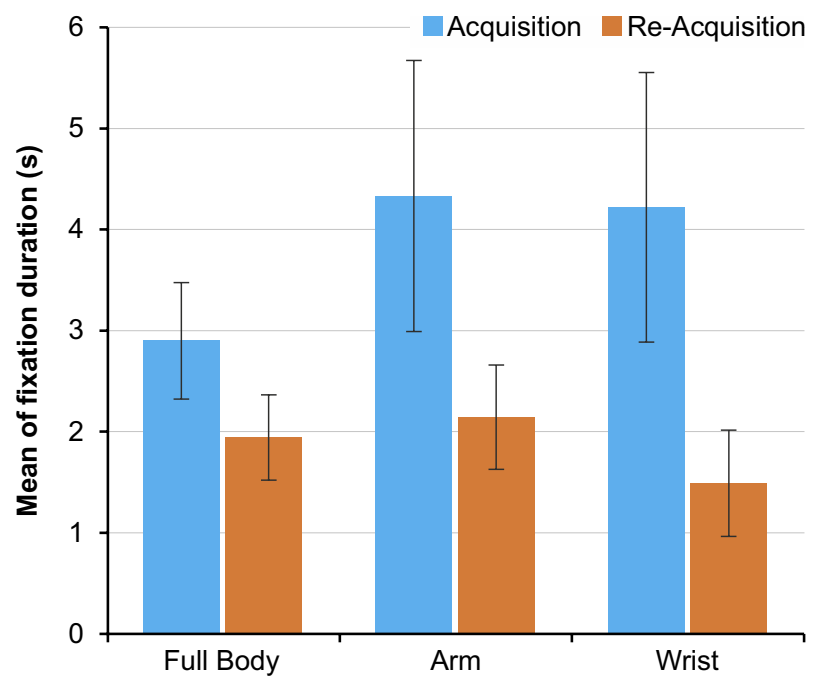

Figure 5. The mean of fixation duration in different test times. Error bars represent standard deviations. 


\section{Discussion}

This research aimed to examine the effect of observing different kinds of information on learning the basketball jump shot. In terms of accuracy, the groups showed progress in the acquisition stage, but there were no significant between-group differences. This was also valid for retention. In re-acquisition, groups made significant progress in the second block compared to the first block, even though this difference was not from the last acquisition block. The results of this research are consistent with some studies conducted in the past (e.g., Breslin et al., 2009; Horn et al., 2005), but inconsistent with others (e.g., Hayes et al., 2007). Several reasons may be responsible for the inconsistencies. First, this can be attributed to scoring system sensitivity. In this research, a Likert scale was used to score jump shots, which had low sensitivity and may not be suitable for showing the differences between groups. Inconsistent studies that show between-group differences (e.g., Hayes et al., 2007) used a metric scale based on distance from the target in centimeters, which is more sensitive than the scale used in the present research. Studies that support the present findings used a similar scale (e.g., Breslin et al., 2005; Breslin et al., 2006, 2009). Another possible reason is the need to achieve the external goal of the task, which may have caused the participants to focus primarily on achieving the goal, thus leading to similar performances (Wohlschläger, Gattis, \& Bekkering, 2003). According to the goal-directed theory of imitation, the observer decomposes the observed action into a hierarchy of goals based on functionality: when a task requires achieving an external goal, the observer prioritizes that goal and imitation of the movement pattern becomes secondary (Wohlschläger et al., 2003). Accordingly, it is possible that the groups studied in this research prioritized the achievement of the task goal, which may have caused similarity in their accuracy. This argument can be tested by studying similar groups without an external goal.

In terms of motor coordination in the acquisition, the performance of the group observing the whole body and the one observing the throwing arm was more similar to the model than the Wrist Group. This effect persisted in during retention. These findings are consistent with some studies in the past (e.g., Breslin et al., 2005, 2006, 2009), but inconsistent with some others (e.g., Hayes et al., 2007; Hodges et al., 2005). Additionally, the results partially support the visual perception perspective (Scully \& Newell, 1985). According to this theory, the observer uses relative motion information to reproduce a movement. From the results of the present findings, displaying relative motion information led to better learning of motor coordination. However, an interesting result was that limited display of relative information (i.e., information from the throwing arm) led to outcomes similar to those of the complete display of relative information. When absolute motion information was displayed, performance suffered. It appears that the amount of information that is displayed is more important than the type of information (Breslin et al., 2009). The amount of information required to reproduce a movement may vary based on the complexity of the task (Breslin et al., 2006, 2009; Hodges, Williams, Hayes, \& Breslin, 2007; Ste-Marie et al., 2012). Studies showing that end-point information can lead to optimal motor learning involved tasks that require movement in one part of the body such as a soccer chip (Hodges et al., 2005). However, in tasks that require full-body movement, such as cricket bowling (Breslin et al., 2006, 2009), observing end-point or absolute motion information leads to poorer learning outcomes than observing relative or limited relative information (Hodges et al., 2007; Ste-Marie et al., 2012). The similarity in the performances of the whole body and throwing hand groups in this research indicates that, in a jump shot, the most important source of information is end-effector (arm) information (i.e., the throwing hand) (Breslin et al., 2009). This argument is further supported by the results of visual search analysis and is explained in the following paragraph.

One of the aims of this research was to determine whether displaying relative information after the early stages of learning has any effect on learning a movement pattern. According to the visual perception perspective (Scully \& Newell, 1985), the observer uses relative information only in the early stages of learning. Consistent with this prediction, the results of the present research showed that displaying relative information to the participants in the re-acquisition stage (even to the group that had no access to relative information in early stages, i.e., the Wrist group) had no significant effect on the similarity of their movement to that of the model. This underlines the importance of the time of presenting the model in the learning process.

Visual search data showed that the Full Body group fixated more on the throwing arm in the acquisition stage, and other groups fixated more on the throwing arm in the re-acquisition stage, even though the time was different. These results are consistent with the results of previous studies (Breslin et al., 2009). As mentioned earlier, the visual search data somehow confirm the kinematic findings. The fact that participants in all the groups focused more on the throwing arm suggests the importance of its information in learning that specific task. Since the Wrist group was deprived of this information in the acquisition stage, their performance may 
have suffered as a result in comparison to the other groups. However, this main source of information was available to the Arm group and thus their performance was not undermined compared to the Full Body group. An important point in these findings is that although after the acquisition stage the absolute motion information group (Wrist) fixated more on the throwing arm, coordination did not improve, which supports the visual perception perspective that relative motion information is effective only at early stages of learning and becomes less and less important at later stages (Scully \& Newell, 1985).

However, even though displaying the relative motion information in the re-acquisition did not improve motor coordination, it did improve the visual search strategy. The results showed that groups had less fixation frequency and fixation duration in the re-acquisition than the acquisition. These changes indicate the release of perceptual degrees of freedom (Breslin et al., 2009). It is believed that observers focus on limited sources of information at the beginning of the learning process and perform frequent, long saccades; however, as time goes on, the observer begins to use other sources of information and spends less time fixating on different areas (Savelsbergh, van der Kamp, Oudejans, \& Scott, 2004). The results support these arguments. As seen from Figures 4 and 5, during re-acquisition, the participants had less fixation frequency and less average fixation time than acquisition, which indicates the release of perceptual degrees of freedom (Breslin et al., 2009; Savelsbergh et al., 2004). As a limitation, the number of participants in each group can influence the results of this study. It is recommended to use more participants in future studies.

\section{Conclusions}

Overall, the results of this research showed that the presentation of relative and limited relative motion information led to better learning of coordination patterns than absolute motion information. Moreover, visual search data indicated that the information from the throwing arm was used by participants when observing a jump shot task. In re-acquisition, displaying the relative information did not improve motor coordination, but did enhance visual search strategy. Consistent with the visual perception perspective, relative motion information (whether displayed completely or in a limited fashion) seems to play a more important role in motor learning than absolute motion information. It seems that the type of task at hand plays an important role in determining the information used in observational learning. These findings can have practical implications for coaches. It is recommended that coaches should focus on displaying relative motion information in the early stages of learning, as this will lead to better learning (at least in terms of motor coordination).

\section{Conflict of interest}

There were no conflicts of interest.

\section{References}

Aiken, C. A., Fairbrother, J. T., \& Post, P. G. (2012). The effects of self-controlled video feedback on the learning of the basketball set shot. Frontiers in Psychology, 3, 338.

Al-Abood, S. A., Davids, K., Bennett, S. J., Ashford, D., \& Marin, M. M. (2001). Effects of manipulating relative and absolute motion information during observational learning of an aiming task. Journal of Sports Sciences, 19, 507-520.

Bandura, A. (1977). Social learning theory. Englewood Cliffs, NJ: Prentice Hall.

Breslin, G., Hodges, N. J., Williams, A. M., Curran, W., \& Kremer, J. (2005). Modelling relative motion to facilitate intra-limb coordination. Human Movement Science, 24, 446-463.

Breslin, G., Hodges, N. J., \& Williams, A. M. (2009). Effect of information load and time on observational learning. Research Quarterly for Exercise and Sport, 80, 480-490.

Breslin, G., Hodges, N. J., Williams, A. M., Kremer, J., \& Curran, W. (2006). A comparison of intra-and inter-limb relative motion information in modelling a novel motor skill. Human Movement Science, 25, 753-766.

Ghorbani, S., \& Bund, A. (2016). Observational learning of a new motor skill: The effect of different model demonstrations. International Journal of Sports Science \& Coaching, 11, 514-522.

Hayes, S. J., Hodges, N. J., Huys, R., \& Williams, A. M. (2007). End-point focus manipulations to determine what information is used during observational learning. Acta Psychologica, 126, 120-137.

Hodges, N. J., Hayes, S. J., Breslin, G., \& Williams, A. M. (2005). An evaluation of the minimal constraining information during observation for movement reproduction. Acta Psychologica, 119, 264-282.

Hodges, N. J., Williams, A. M., Hayes, S. J., \& Breslin, G. (2007). What is modelled during observational learning? Journal of Sports Sciences, 25, 531-545.

Horn, R. R., Williams, A. M., \& Scott, M. A. (2002). Learning from demonstrations: The role of visual search during observational learning from video and point-light models. Journal of Sports Sciences, 20, 253-269.

Horn, R. R., Williams, A. M., Scott, M. A., \& Hodges, N. J. (2005). Visual search and coordination changes in response to video and point-light demonstrations without KR. Journal of Motor Behavior, 37, 265-274.

Johansson, G. (1973). Visual perception of biological motion and a model for its analysis. Perception \& Psychophysics, 14, 201-211. 
Romack, J. L. (1995). Information in visual event perception and its use in observational learning. In B. G. Bardy, R. J. Bootsma, \& Y. Guiard (Eds.), Studies in perception and action III (pp. 289-292). London, United Kingdom: Routledge.

Savelsbergh, G. J. P., van der Kamp, J., Oudejans, R. R. D., \& Scott, M. A. (2004). Perceptual learning is mastering perceptual degrees of freedom. In A. M. Williams \& N. J. Hodges (Eds.), Skill acquisition in sport: Research, theory and practice (pp. 374-389). London, United Kingdom: Routledge.

Scully, D., \& Carnegie, E. (1998). Observational learning in motor skill acquisition: A look at demonstrations. Irish Journal of Psychology, 19, 472-485.

Scully, D. M., \& Newell, K. M. (1985). Observational learning and the acquisition of motor skills: Toward a visualperception perspective. Journal of Human Movement Studies, 11, 169-186.
Sidaway, B., Heise, G., \& Schoenfelder-Zohdi, B. (1995). Quantifying the variability of angle-angle plots. Journal of Human Movement Studies, 29, 181-197.

Ste-Marie, D. M., Law, B., Rymal, A. M., Jenny, O., Hall, C., \& McCullagh, P. (2012). Observation interventions for motor skill learning and performance: An applied model for the use of observation. International Review of Sport and Exercise Psychology, 5, 145-176.

Williams, A. M., Davids, K., \& Williams, J. G. P. (1999). Visual perception and action in sport. London, United Kingdom: Routledge.

Winter, D. A. (1990). Biomechanics and motor control of human movement (2nd ed.). New York, NY: Wiley.

Wohlschläger, A., Gattis, M., \& Bekkering, H. (2003). Action generation and action perception in imitation: An instance of the ideomotor principle. Philosophical Transactions of the Royal Society of London. Series B: Biological Sciences, 358, 501-515. 